\title{
Pentoxifylline Prevents Driamycin-Induced Myocardial Fibrosis and Apoptosis in Rats
}

\author{
Zhijun ZANG, ${ }^{1,2} \mathrm{PhD}$, Shujuan $\mathrm{LI},{ }^{3} \mathrm{PhD}$, Yuese $\mathrm{Lin},{ }^{3} \mathrm{MD}$, Xuandi LI, ${ }^{3} \mathrm{MD}$, Yunquan $\mathrm{LI},{ }^{3} \mathrm{PhD}$, \\ Youzhen QIN, ${ }^{3} \mathrm{PhD}$, Huishen WANG, ${ }^{3} \mathrm{MD}$, Meihua JiANG,${ }^{2,4} \mathrm{PhD}$, and Ling ZhU, ${ }^{3} \mathrm{PhD}$
}

\begin{abstract}
SUMMARY
Adriamycin (ADR) is a potent antineoplastic agent, but long-term treatment is limited by its cumulative, life-threatening cardiomyopathy. Recently, a few reports have shown that pentoxifylline (PTX) might produce cardioprotection in cardiac dysfunction. Here, we investigated the protective effects of PTX on ADR-induced cardiomyopathy in rats. Male rats were randomly assigned either to saline, ADR (adriamycin, $5 \mathrm{mg} / \mathrm{kg} / \mathrm{week}$ ), or A (adriamycin, $5 \mathrm{mg} / \mathrm{kg} / \mathrm{week}$ ) + PTX (pentoxifylline, $50 \mathrm{mg} / \mathrm{kg} / \mathrm{day}$ ) groups. After 3 weeks, these animals were sacrificed and the heart tissue was harvested for histological analysis and assessment of hepatocyte growth factor (HGF) and caspase-3 expression. Histopathological findings showed that PTX can alleviate myocardial damage caused by ADR. Cardiac fibrosis was significantly suppressed in the A+PTX group compared to that in the ADR group. The HGF gene expression was decreased significantly in the ADR group compared with the control group, but was increased in the A+PTX group. Caspase-3 was up-regulated in the ADR group, and down-regulated in the A+PTX group. These results show that treatment with PTX exerts a protective effect against ADR-induced myocardial fibrosis via regulation of HGF and caspase-3 gene expression. PTX may thus represent a useful new clinical tool for the treatment of ADR-induced cardiomyopathy. (Int Heart J 2015; 56: 651-655)
\end{abstract}

Key words: Antineoplastic, Cardiomyopathy

A driamycin (ADR), an anthracycline drug, has been established as an effective agent against various kinds of solid tumors and hematologic malignancies. ${ }^{1)}$ However, its clinical use is limited by its dose-dependent cardiotoxicity and irreversible cardiomyopathy which result in dilated cardiomyopathy (DCMP) with congestive heart failure. ${ }^{2)}$ The mechanism of ADR-induced DCMP is thought to be the up-regulation of reactive free oxygen radicals which can make the heart vulnerable to injury through lowering the activities of protective enzymes such as topoisomerase II and ghrelin. ${ }^{3-5)}$ The fibrotic mediators play an important role in the pathogenesis of DCMP induced by ADR. Hepatocyte growth factor (HGF), a well-known multifunctional cytokine, was shown to be involved in cardiac fibrotic processes and played an important role in anti-fibrosis during the course of DCMP ${ }^{6-8)}$ These reports also showed that phosphodiesterases (PDEs) remarkably express in cardiomyocyte apoptosis, and can induce cAMP and cGMP-dependent apoptosis in cardiomyopathy. ${ }^{6-8)}$ In this pathway, caspases are crucial mediators. As critical participators in the orchestration of apoptosis, caspases cleave the target proteins to execute cell death. Among them, caspase- 3 is the most important one. It can target structural substrates including nuclear laminins, focal adhesion sites, and cell-cell adherence junctions, and catalyze the specific cleavage of many key cellular proteins. ${ }^{9-15)}$

Pentoxifylline (PTX) is a methylxanthine derivative and a nonspecific PDE inhibitor which has been used for decades. In recent years, many studies have showed that PTX can decrease the level of inflammatory mediators through blockade of the expression of inflammatory genes, and thereby reduce the generation of inflammatory factor. ${ }^{16-18)}$ The present study was designed to show the expression of HGF and caspase- 3 in this ADR-induced cardiomyopathy after the administration of PTX in order to explore the mechanism of PTX acting on DCMP.

\section{Methods}

Animals and experimental protocol: Thirty male SpragueDawley rats (6 weeks old, 200 220 g) were purchased from the Animal Center of Sun Yat-sen University (Guangzhou, China) and housed individually in cages at constant tempera-

From the ${ }^{1}$ Department of Infertility and Sexual Medicine, The Third Affiliated Hospital, ${ }^{2}$ Key Laboratory for Stem Cells and Tissue Engineering, Center for Stem Cell Biology and Tissue Engineering, Ministry of Education, ${ }^{3}$ Department of Pediatric Cardiology, The First Affiliated Hospital, and ${ }^{4}$ Department of Anatomy, Zhongshan School of Medicine, Sun Yat-sen University, Guangzhou, Guangdong, China.

This work was supported by the National Natural Science Foundation of China (grant no. 81200945), and the Fundamental Research Funds for the Central Universities (grant no.14ykpy01).

Address for correspondence: Meihua Jiang, PhD, Key Laboratory for Stem Cells and Tissue Engineering, Center for Stem Cell Biology and Tissue Engineering, Ministry of Education, Sun Yat-sen University, Guangzhou, Guangdong 510080, China. E-mail: jiangmeihua1977@ 163.com or Ling Zhu, PhD, Department of Pediatric Cardiology, The First Affiliated Hospital, Sun Yat-sen University, Guangzhou, Guangdong 510080, China. E-mail: zhulingguangzhou@163.com

Received for publication May 27, 2015. Revised and accepted June 18, 2015.

Released in advance online on J-STAGE November 6, 2015.

All rights reserved by the International Heart Journal Association. 
ture $\left(22 \pm 1^{\circ} \mathrm{C}\right)$ and humidity $(60 \%)$. Animal and experiment protocols were conducted in accordance with the NIH Guidelines for the Care and Use of Laboratory Animals and were approved by the Sun Yat-sen University Institutional Animal Care and Use Committee. All of the rats were randomly divided into 3 equal groups ( $n=10$ per group). In the control group, animals were treated with saline. In the ADR group, animals were injected with $\mathrm{ADR}$ at a dose of $5 \mathrm{mg} / \mathrm{kg} /$ week iv; In the A+PTX group, animals were also injected with ADR at a dose of $5 \mathrm{mg} / \mathrm{kg} /$ week iv and received PTX at a dosage of $50 \mathrm{mg} /$ $\mathrm{kg} /$ day by gavage through a gastric needle. On day 21 , all animals were sacrificed by cervical dislocation under ether anesthesia. The hearts were removed and subjected to further analysis.

Pathological evaluation of the heart tissue: Heart tissues were immediately fixed in $4 \%$ paraformaldehyde (PFA) for 24 hours and embedded in paraffin. Subsequently, heart tissues were sectioned at a thickness of $5 \mu \mathrm{m}$ and stained with Masson's-trichrome. Ten microscopic fields of blinded sections were randomly selected and analyzed using a color image analyzer (BX-51/Image-Pro plus, Olympus, Tokyo). To evaluate fibrotic changes of the heart, tissue sections were stained with Massontrichrome. The fibrotic area of the total area was determined by computer assisted analysis in each tissue section. At least 10 fields at 200-fold magnification were captured and assessed in all the samples. The fibrosis extent and myocyte diameter in these photomicrographs was quantified by a blinded observer using the Image J program from NIH Image Software (National Institutes of Health, Bethesda, MD, USA). All data were analysed in a blind fashion by two independent investigators and then averaged.

Reverse transcription-polymerase chain reaction (RT-PCR): Total RNA from the heart tissue was extracted using an RNeasy mini kit (Qiagen Ltd, UK) according to the manufacturer's protocol. Reverse transcription reactions were performed using murine leukemia virus reverse transcriptase and oligo-dT primers (Fermentas, Ltu). Specific primer sets for HGF and GAPDH used for RT-PCR analysis were: HGF forward, 5'ACCAAGGAAGACCCATTACTGAAGA; HGF reverse, 5'TTCCAAGGCTGGCATTTGATGC; GAPDH forward, 5'ACCACAGT CCATGCCATCAC; GAPDH reverse, 5'TCCACCACCCTGTTGCTGTA.

Western blot analysis: Separated heart tissues were powdered in liquid nitrogen before protein was isolated and suspended in radioimmunoprecipitation assay lysis buffer (Sigma Chemical Co., St Louis, USA). The homogenates were centrifuged at $10,000 \times \mathrm{g}$ for 5 minutes at $4^{\circ} \mathrm{C}$. The protein in supernatant was determined using a Bio-Rad DC protein assay (Bio-Rad Laboratories, Hercules, CA, USA). Next, $20 \mu \mathrm{g}$ of each protein was administered separately to SDS-PAGE and transferred to PVDF membranes. After blocking with 5\% nonfat dry milk in TBST $(0.1 \%$ Tween $20,100 \mathrm{mM} \mathrm{NaCl}$, and $10 \mathrm{mM}$ Tris-HCl, $\mathrm{pH}$ 7.4) for 1 hour, the membranes were incubated with polyclonal primary antibody HGF and caspase-3 (1: 100, both, Cell Signaling Technology, USA) overnight at $4^{\circ} \mathrm{C}$. After washing in TBST 3 times, the membranes were incubated with HRPconjugated secondary antibody (1:3000, Vector, Burlingame, USA) for 2 hours at room temperature. Signals were visualized using an enhanced chemiluminescence reagent (Amersham Bioscience, Uppsala, Sweden).

Apoptosis assay: Apoptotic nuclei were detected using termi- nal deoxynucleotidyl transferase mediated dUTP Nick End Labeling (TUNEL) staining kits (Roche Applied Science, Indianapolis, IN) according to the manufacturer's instructions. Images were taken in 10 random fields (magnification X400) for each animal. Nuclei were counterstained with hematoxylin (blue). The numbers of TUNEL-positive cells and total cardiomyocyte nuclei were counted, and the apoptotic index of cardiomyocytes was expressed as the percentage of TUNEL-positive cells to total cells. The results are expressed as the percentages of apoptotic cell numbers in each field.

Statistical analysis: All data are expressed as the mean \pm SEM. Statistical differences between different groups were analyzed using one-way analysis of variance (ANOVA) with Newman-Keuls post hoc comparison. Values of $P<0.05$ were considered significant.

\section{RESUlTS}

Histological analysis of the heart after ADR treatment: Fibrosis has been suggested to be involved in cardiac stiffness and dysfunction in ADR-induced cardiotoxicity. ${ }^{19)}$ To explore the cardiac pathological changes of fibrosis, we performed Masson's trichrome staining after ADR administration in each animal group. Cardiomyopathy induced by ADR treatment was exacerbated in rats. There were no deaths and no signs of overt toxicity during the study. The histochemical features of myocardium in each group were examined in the fibrotic areas observed through microscopy. In the ADR group, the histological results showed that ADR injection gave rise to myocardial damage, such as myofibrillar loss, contraction band necrosis, and fibrosis after tissue apoptosis. However, these phenomena were alleviated when treated with PTX (Supplemental Figure 1A). The area of fibrosis seen on Masson's trichrome staining significantly increased in the ADR group compared with the A+PTX group $(21.08 \% \pm 2.28 \%$ versus $10.27 \% \pm 3.28 \%, P<$ 0.05) (Supplemental Figure 1B).

PTX effect on HGF gene expression after ADR induced cardiomyopathy: HGF is an anti-fibrosis cytokine. The expression of HGF mRNA was significantly decreased in the ADR group compared with the control group $(P<0.05)$. Moreover, the HGF mRNA expression was increased 2- to 3-fold in the A+PTX group compared to the ADR group $(P<0.01)$ (Figure $1 \mathrm{~A}$ and $\mathrm{B})$. Similarly, the expression of HGF protein was also assessed and found to be decreased in the ADR group and in $40 \%$ of control specimens, but increased after PTX treatment (Figure 1C and D).

PTX administration inhibits myocardial apoptosis after ADR induced cardiomyopathy: HGF prohibits apoptotic signals via inhibition of caspase-3 activity. ${ }^{20)}$ In this research, the protein expression of caspase- 3 was also evaluated by Western blot analysis in each group. As shown in Figure 2, caspase-3 was found to be up-regulated in the ADR group $(P<0.01)$. After treatment with PTX, the expression of caspase-3 protein was significantly down-regulated $(P<0.05)$. We also performed TUNEL staining to assess the effect of PTX on ADR-treated cardiomyocyte apoptosis (Supplemental Figure 2). Compared with the control group, the percentage of apoptotic cells was increased in the cardiomyocytes of the ADR group (Figure 3). Remarkably, the rats treated with PTX showed a marked decrease in apoptotic cell death compared to the ADR treated rats 

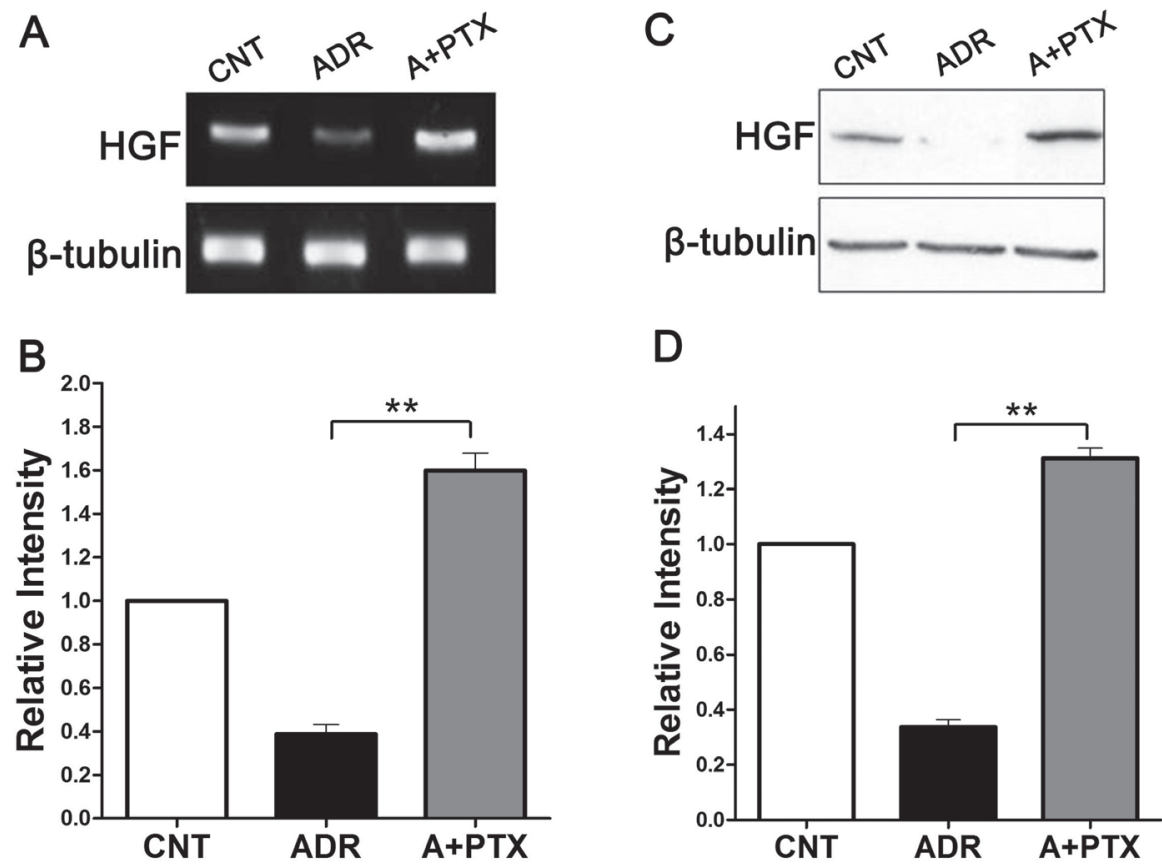

Figure 1. Expression of HGF in each group at day 21. A: The PCR original figure. The ADR group showed decreased HGF mRNA expression compared with the control group, and the A+PTX group showed higher HGF mRNA expression than the ADR group. B: The histogram of relative intensity derived from A. The relative value of HGF mRNA was also higher in the A+PTX group. C: The level of HGF protein decreased in the ADR group but increased in the A+PTX group. D: The histogram of relative intensity derived from $\mathbf{C}$. The relative value of HGF protein was also higher in the A+PTX group than in the ADR group. Values are presented as the mean \pm SEM. ${ }^{* *} P<0.01, n=10$ per group.

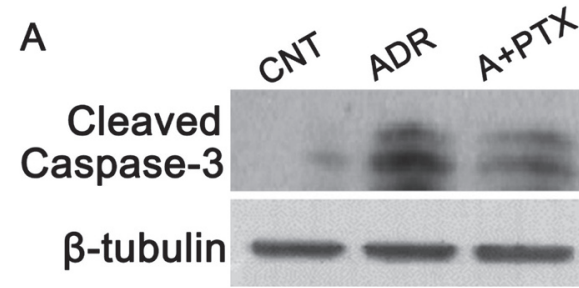

B

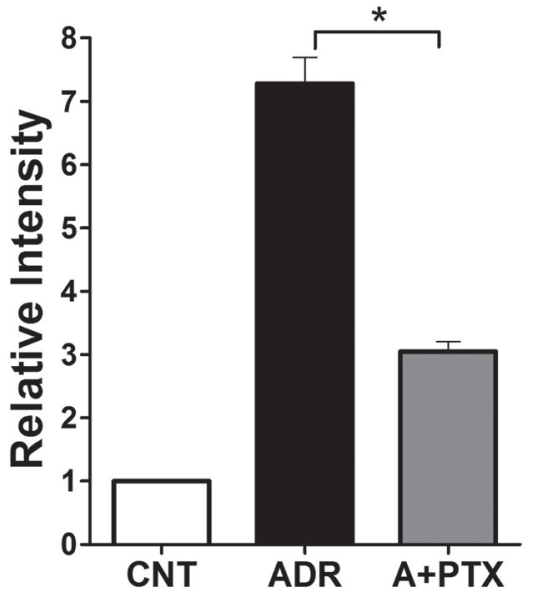

Figure 2. Outcome of Western blot and relative intensity analysis of caspase-3 protein expression in the myocardium of each group at day 21 . A: The caspase-3 expression was higher in the ADR group, but lower in the A+PTX group. B: The histogram of relative intensity further demonstrated that the concentration of caspase- 3 protein increased in the ADR group but significantly decreased in the A+PTX group. Data are expressed as the mean \pm SEM. ${ }^{*} P<0.05, n=10$ per group.

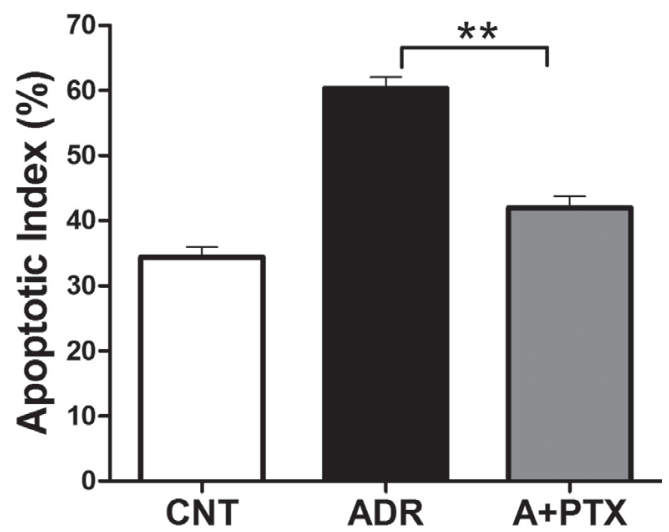

Figure 3. The apoptotic index of cardiomyocytes of the rats in each group. Quantitative analysis showed that the apoptotic index of cardiomyocytes was much higher in the ADR group than the other two groups. Values are presented as the mean \pm SEM. $^{* * *} P<0.01, n=10$ per group.

$(P<0.01)$ (Figure 3).

\section{Discussion}

The results of the present study have demonstrated that PTX has a protective effect on injury to the heart in ADRtreated rats. ADR treatment was significantly associated with increased myocardial fibrosis and apoptosis. However, PTX treatment could regulate HGF gene expression and decrease cardiac fibrosis and down regulate caspase- 3 mediated myo- 
cardial apoptosis.

Adriamycin is one of the most effective and useful antineoplastic agents for the treatment of various malignancies. However, its practical therapeutic use is sometimes limited by the frequent induction of acute cardiotoxicity. ${ }^{21,22)}$ These cellular mechanisms, including myocardial fibrosis, apoptosis, and altered energy metabolism, have been proposed to account for the cardiomyopathy caused by ADR..$^{19,23,24)}$

Fibrosis has been suggested to be involved in cardiac stiffness and dysfunction, which are caused when increased collagen synthesized by the fibroblasts invades and replaces necrotic or apoptotic myocytes. ${ }^{19)}$ In this study, we observed that ADR-induced fibrosis was prevented by PTX, as presented by the attenuation of collagen deposition. PTX, a phosphodiesterase inhibitor, has a clinical application as an inhibitor of platelet aggregation. Several studies imply that its cellular effects also include anti-fibrotic effects in a variety of mesenchymal cells. ${ }^{25-28)}$ Furthermore, PTX administration has been shown to inhibit mitogenesis and collagen synthesis in renal fibroblast cultures. ${ }^{27,28)}$ Here, our study found an increased expression of myocardial HGF mRNA and protein occurred after the administration of PTX. HGF is a well-known anti-fibrosis factor in the development of heart disease. In the protection process, HGF can significantly reduce the generation of extracellular matrix, increase the production of matrix metalloprotease- 1 and urokinase plasminogen, as well as decrease TGF- $\beta$ concentration. $^{8,29,30)}$ TGF- $\beta$, which is a pathogenic factor that stimulates fibrosis through the accumulation of extracellular matrix, is up-regulated in cardiomyopathy. Moreover, PTX could improve liver regeneration via down-regulation of TGF- $\beta 1$ gene expression. ${ }^{31)}$ Thus, PTX may have improved the cardiac function by up-regulated HGF, which functions through further suppressing the expression of TGF- $\beta$. The exact mechanisms remain unclear and need further study.

ADR exposure causes activation of mitochondrial apoptosis in cardiomyocytes. ${ }^{32}$ The caspase cascade induction is considered one of the mechanisms of intensification of apoptosis in cardiomyocytes, which is manifested in increased activity of the caspase- 3 effector caspase in the heart. ${ }^{33)}$ Here, we also confirmed that ADR significantly induces myocardium apoptosis and increases caspase- 3 activity. Compared with ADR-treated rats, PTX-treated rats underwent significantly less histologic change of myocardial tissue, and had a smaller apoptotic index, including lower TUNEL positive cells and down regulated caspase- 3 expression. A number of previous studies have also demonstrated that HGF exerted an anti-apoptotic effect through the proapoptotic caspase-3 signaling pathway in various cells. ${ }^{34,35)}$ It has been reported that PTX can significantly decrease caspase-3 activities and reduce apoptosis to a significant extent in rat pups with hypoxic-ischemic encephalopathy. ${ }^{36)}$ Collectively, our data suggest that PTX prevents the activation of myocardial apoptosis induced by ADR, and the anti-apoptotic effect of PTX is probably mediated through cellular signaling of the HGF pathways.

According to the results of our research, PTX treated rats exhibited significantly attenuated cardiac fibrotic changes from macro (histopathology) and micro (cytokine) aspects. Other histopathological improvements also occurred, including significant decreases in necrosis, interstitial fibrosis, and apoptosis. Therefore, PTX might reduce DCMP occurrence, which is an end-stage cardiomyopathic heart disease.
In conclusion, PTX might be effective in the treatment of ADR-induced cardiomyopathy. Our research implies that PTX can play an important role in relieving the cardiotoxicity due to a high cumulative dose of ADR. Furthermore, the cardio-protective effect of this drug may occur through blocking the caspase3-dependent apoptotic pathway and may create factors that play anti-apoptotic roles. Moreover, we found that PTX treatment significantly increased HGF, the anti-fibrotic cytokine, which plays an important role in reversing the process of heart disease. In summary, our results suggest that cardioprotection of ADR-induced cardiomyopathy can be improved by treatment with PTX.

\section{REFERENCES}

1. Singal PK, Iliskovic N. Doxorubicin-induced cardiomyopathy. N Engl J Med 1998; 339: 900-5. (Review)

2. Grenier MA, Lipshultz SE. Epidemiology of anthracycline cardiotoxicity in children and adults. Semin Oncol 1998; 25: 72-85. (Review)

3. Gewirtz DA. A critical evaluation of the mechanisms of action proposed for the antitumor effects of the anthracycline antibiotics adriamycin and daunorubicin. Biochem Pharmacol 1999; 57: 72741. (Review)

4. Keizer HG, Pinedo HM, Schuurhuis GJ, Joenje H. Doxorubicin (adriamycin): a critical review of free radical-dependent mechanisms of cytotoxicity. Pharmacol Ther 1990; 47: 219-31. (Review)

5. Xu Z, Wu W, Lan T, Zhang X. Protective effects of extract of Ginkgo biloba on adriamycin-induced heart failure and its mechanism: role of ghrelin peptide. Zhongguo Zhong Yao Za Zhi 2009; 34: 2786-9. (Chinese)

6. Blobe GC, Schiemann WP, Lodish HF. Role of transforming growth factor beta in human disease. N Engl J Med 2000; 342: 1350-8. (Review)

7. Chen MM, Lam A, Abraham JA, Schreiner GF, Joly AH. CTGF expression is induced by TGF-beta in cardiac fibroblasts and cardiac myocytes: a potential role in heart fibrosis. J Mol Cell Cardiol 2000; 32: 1805-19.

8. Taniyama Y, Morishita R, Nakagami H, et al. Potential contribution of a novel antifibrotic factor, hepatocyte growth factor, to prevention of myocardial fibrosis by angiotensin II blockade in cardiomyopathic hamsters. Circulation 2000; 102: 246-52.

9. Porter AG, Jänicke RU. Emerging roles of caspase-3 in apoptosis. Cell Death Differ 1999; 6: 99-104. (Review)

10. Brancolini C, Lazarevic D, Rodriguez J, Schneider C. Dismantling cell-cell contacts during apoptosis is coupled to a caspase-dependent proteolytic cleavage of beta-catenin. J Cell Biol 1997; 139: 759-71.

11. Kook S, Kim DH, Shim SR, Kim W, Chun JS, Song WK. Caspase-dependent cleavage of tensin induces disruption of actin cytoskeleton during apoptosis. Biochem Biophys Res Commun 2003; 303: 37-45.

12. Taylor RC, Cullen SP, Martin SJ. Apoptosis: controlled demolition at the cellular level. Nat Rev Mol Cell Biol 2008; 9: 231-41. (Review)

13. Thornberry NA, Lazebnik Y. Caspases: enemies within. Science 1998; 281: 1312-6. (Review)

14. Kothakota S, Azuma T, Reinhard C, et al. Caspase-3-generated fragment of gelsolin: effector of morphological change in apoptosis. Science 1997; 278: 294-8.

15. Timmer JC, Salvesen GS. Caspase substrates. Cell Death Differ 2007; 14: 66-72. (Review)

16. Waage A, Sørensen M, Størdal B. Differential effect of oxpentifylline on tumour necrosis factor and interleukin-6 production. Lancet 1990; 335: 543.

17. Sliwa K, Skudicky D, Candy G, Wisenbaugh T, Sareli P. Ran- 
domised investigation of effects of pentoxifylline on left-ventricular performance in idiopathic dilated cardiomyopathy. Lancet 1998; 351: 1091-3.

18. Mann DL. Inflammatory mediators and the failing heart: past, present, and the foreseeable future. Circ Res 2002; 91: 988-98. (Review)

19. Miyata S, Takemura G, Kosai K, et al. Anti-Fas gene therapy prevents doxorubicin-induced acute cardiotoxicity through mechanisms independent of apoptosis. Am J Pathol 2010; 176: 687-98.

20. Xiao GH, Jeffers M, Bellacosa A, Mitsuuchi Y, Vande WGF, Testa JR. Anti-apoptotic signaling by hepatocyte growth factor/Met via the phosphatidylinositol 3-kinase/Akt and mitogen-activated protein kinase pathways. Proc Natl Acad Sci U S A 2001; 98: 247-52.

21. Hequet O, Le QH, Moullet I, et al. Subclinical late cardiomyopathy after doxorubicin therapy for lymphoma in adults. J Clin Oncol 2004; 22: 1864-71.

22. Lipshultz SE, Rifai N, Dalton VM, et al. The effect of dexrazoxane on myocardial injury in doxorubicin-treated children with acute lymphoblastic leukemia. N Engl J Med 2004; 351: 145-53.

23. Carvalho RA, Sousa RP, Cadete VJ, et al. Metabolic remodeling associated with subchronic doxorubicin cardiomyopathy. Toxicology 2010; 270: 92-8.

24. Sharma H, Pathan RA, Kumar V, Javed S, Bhandari U. Anti-apoptotic potential of rosuvastatin pretreatment in murine model of cardiomyopathy. Int J Cardiol 2011; 150: 193-200.

25. Windmeier C, Gressner AM. Pharmacological aspects of pentoxifylline with emphasis on its inhibitory actions on hepatic fibrogenesis. Gen Pharmacol 1997; 29: 181-96. (Review)

26. Tsai TJ, Lin RH, Chang CC, et al. Vasodilator agents modulate rat glomerular mesangial cell growth and collagen synthesis. Nephron 1995; 70: 91-9.

27. Hewitson TD, Martic M, Kelynack KJ, Pedagogos E, Becker GJ Pentoxifylline reduces in vitro renal myofibroblast proliferation and collagen secretion. Am J Nephrol 2000; 20: 82-8.

28. Strutz F, Heeg M, Kochsiek T, Siemers G, Zeisberg M, Müller GA. Effects of pentoxifylline, pentifylline and gamma-interferon on proliferation, differentiation, and matrix synthesis of human renal fibroblasts. Nephrol Dial Transplant 2000; 15: 1535-46.

29. Nakamura T, Matsumoto K, Mizuno S, Sawa Y, Matsuda H, Na- kamura T. Hepatocyte growth factor prevents tissue fibrosis, remodeling, and dysfunction in cardiomyopathic hamster hearts. Am J Physiol Heart Circ Physiol 2005; 288: H2131-9.

30. Yi X, Li X, Zhou Y, et al. Hepatocyte growth factor regulates the TGF- $\beta 1$-induced proliferation, differentiation and secretory function of cardiac fibroblasts. Int J Mol Med 2014; 34: 381-90.

31. Martino RB, Coelho AM, Kubrusly MS, et al. Pentoxifylline improves liver regeneration through down-regulation of TNF- $\alpha$ synthesis and TGF- $\beta 1$ gene expression. World J Gastrointest Surg 2012; 4: 146-51.

32. Childs AC, Phaneuf SL, Dirks AJ, Phillips T, Leeuwenburgh C. Doxorubicin treatment in vivo causes cytochrome $\mathrm{C}$ release and cardiomyocyte apoptosis, as well as increased mitochondrial efficiency, superoxide dismutase activity, and Bcl-2:Bax ratio. Cancer Res 2002; 62: 4592-8.

33. Reeve JL, Szegezdi E, Logue SE, et al. Distinct mechanisms of cardiomyocyte apoptosis induced by doxorubicin and hypoxia converge on mitochondria and are inhibited by Bcl-xL. J Cell Mol Med 2007; 11: 509-20.

34. Kitamura K, Iwanami A, Nakamura M, et al. Hepatocyte growth factor promotes endogenous repair and functional recovery after spinal cord injury. J Neurosci Res 2007; 85: 2332-42.

35. Liu Y, Shi QF, Qi M, Tashiro S, Onodera S, Ikejima T. Interruption of hepatocyte growth factor signaling augmented oridonin-induced death in human non-small cell lung cancer A549 cells via c-metnuclear factor- $\kappa \mathrm{B}$-cyclooxygenase- 2 and $\mathrm{c}-\mathrm{Met}-\mathrm{Bcl}-2$-caspase-3 pathways. Biol Pharm Bull 2012; 35: 1150-8.

36. Kalay S, Oztekin O, Tezel G, et al. The effects of intraperitoneal pentoxifylline treatment in rat pups with hypoxic-ischemic encephalopathy. Pediatr Neurol 2013; 49: 319-23.

\section{SUPPLEMENTAL Files}

Supplemental Figure 1, 2

Please find supplemental files;

https://www.jstage.jst.co.jp/article/ihj/56/6/56_15-203/_article/supplement 